\title{
Commentary on the draft revised guideline on the environmental risk assessment of medicinal products for human use
}

\author{
Rhys Whomsley ${ }^{*}$, Susanne Brendler-Schwaab², Eadaoin Griffin ${ }^{3}$, John Jensen ${ }^{4,5}$, Caroline Moermond ${ }^{6}$, \\ Birger Scholz ${ }^{7}$, Laila Sortvik Nilssen ${ }^{8}$, Henry Stemplewski ${ }^{9}$ and Ines Roennefahrt ${ }^{10}$
}

\begin{abstract}
Applicants for marketing authorisation for human medicinal products in the European Union must submit an environmental risk assessment which is assessed by assessors from the national competent authorities. The EMA guideline on the environmental risk assessment of medicinal products for human use came into effect on 1 December 2006. After 12 years' experience with the guideline, the EMA has released for public consultation a draft revision of the guideline. The revision proposes significant substantive and structural changes to the guideline. The major changes proposed in the revision are outlined together with the rationale for the changes and the expected impact on stakeholders.
\end{abstract}

Keywords: Environmental risk assessment, European Medicines Agency, Human pharmaceuticals, ERA, EMA, CHMP, PBT

\section{Background}

The European Medicines Agency (EMA) has released for public consultation a draft revision of the guideline on the environmental risk assessment (ERA) of medicinal products for human use [1]. The current version of the guideline [2], which came into effect on 1st June 2006, describes how the ERA should be performed by applicants during the application for marketing authorization of human medicinal products (HMP), although a risk to the environment cannot be considered as a criterion to refuse a marketing authorisation. The current guideline describes a tiered testing strategy recommended for the evaluation process. In addition, there is a questions and answer (Q \& A) document [3] to the guideline, released in 2011 and updated in 2016, which provided additional details to the guideline and expanded on areas including ERA for generic medicines, persistent, bioaccumulative and toxic substance (PBT) assessment, endocrine disrupting compounds and metabolites. Substantial evidence and expertise on the ERA for human medicinal

\footnotetext{
${ }^{*}$ Correspondence: Rhys.Whomsley@ema.europa.eu

${ }^{1}$ European Medicines Agency (EMA), Amsterdam, The Netherlands

Full list of author information is available at the end of the article
}

products have been gathered in the EU network, since the introduction of the guideline in 2006. This expertise, together with the need to integrate into the guideline the content of the Q \& A document developed in support of the guideline since 2006, was the trigger for the revision.

In 2016, the EMA published for public consultation a concept paper [4] in support of the revision of the guideline which documented 8 proposed issues for revision, including the tiered approach strategy and relevant triggers for progressing through the tiers, better utilization of publicly available data, assessment approaches for PBT substances and endocrine disruptors, updates on test systems/assays and options for risk mitigation measures. After a consultation period, comments were received from 8 parties (industry, regulators, lobby groups and contract research organizations). The revision to the guideline also builds on changes in relevant guidance documents under other legislative frameworks, advances in knowledge of the effects of pharmaceuticals on the environment from the scientific literature and evaluation of the performance of the present guideline in relation to the new scientific information. Finally, in the interest of animal welfare, the guideline encourages the implementation of the principles of 3Rs (Replacement, Reduction 
and Refinement) in accordance with Directive 2010/63/ EU [5].

This commentary will outline the proposed changes and their expected impact on the conduct of ERAs for human medicinal products (HMP).

\section{Guideline revision}

\section{Decision tree approach}

The revised GL complies with the legislation in Directive 2001/83/EC [6], which requires an ERA for all applications for marketing authorisation approval. The ERA is a tiered approach consisting of two parts:

1. A mandatory phase I assessment based on environmental exposure and general characteristics of the HMP.

2. A phase II assessment in which experimental studies need to be conducted for a detailed fate and effects assessment.

Central to phase I is a decision tree which identifies substances for which a phase II ERA is required. The decision tree incorporates elements from the current guideline and gives a visual guidance. The revision proposes the same threshold value for predicted environmental concentration in surface water $\left(\mathrm{PEC}_{\mathrm{SW}}\right)$ $(0.01 \mu \mathrm{g} / \mathrm{L})$ to decide if the active substance should proceed to the fate and effects assessment. For already approved substances exceeding the threshold value, the current guideline allows the applicant to justify the absence of a complete ERA by demonstrating the absence of a significant increase of the environmental exposure, for example, by providing the consumption data of the active ingredient. This option is not carried forward into the revision in the case of a new application for an approved substance for which an applicant does not have access to the original ERA or for which an ERA is not available. This is because it is not possible to assess that substance's risks on the environment, which is essential information for stakeholders including consumers, environmental scientists, sewage treatment plant managers and water suppliers. In case an application is based on an approved substance for which ERA data are already available, at present, cross reference to proprietary ERA data without a letter of consent is not possible. The revision encourages sharing of data between applicants. This is in the interest of 3Rs and animal welfare, in accordance with the extended ERA concept proposed by industry [7]. The approach to ERA in the decision tree is expected to result in equal requirements for all applicants.

\section{Search and evaluation of data}

As the marketing authorisation is product based and not substance based, for each product, a targeted ERA should be performed. In certain cases, where the assessment stops in phase I, the ERA may consist of a statement that no further assessment is necessary. However, when a phase II assessment needs to be performed, for active substances that are already marketed, information may be available in the public domain. The current guideline states that all relevant data should be taken into account in the phase II assessment. The revision further specifies that a complete review of the published experimental physico-chemical, fate and ecotoxicological studies is required. Specific guidance on search, use and evaluation of published data is provided in the revised guideline. These are aimed at preventing repetition of (animal) studies. When other marketing authorisation holders have already performed relevant studies, the revision encourages mutual sharing of data to minimize the number of tests having to be re-performed. These data can then be used with a letter of access. All data submitted (whether study reports or peer reviewed literature) should contain enough information to permit assessment of the reliability of the study performed.

\section{Tailored assessment for active substances with a specific mode of action}

The revised guideline describes a tailored assessment for certain groups of active substances due to their specific mode of action. The tailored assessment is necessary to ensure that the most sensitive and appropriate tests with specific groups of organisms are used in the assessment. The tailored assessment concerns compounds for which the action limit does not apply, such as endocrine active substances (EAS), but may also concern compounds for which the action limit applies, such as antibiotics. For EAS, a phase II ERA always needs to be performed, regardless of whether their $\mathrm{PEC}_{\mathrm{SW}}$ meets the trigger value of $0.01 \mu \mathrm{g} / \mathrm{L}$. For these substance classes, the tailored approach is described (or expanded for EAS) and waivers for some studies are proposed. For instance, in the case of antibiotics, scientific knowledge and empirical data have demonstrated that a tailored risk assessment focused on the effects on lower trophic levels including bacteria, algae and aquatic invertebrates is sufficiently sensitive for antibiotics and therefore fish tests are not required. The identification of EAS has been broadened compared to the current guideline, which only focuses on potential sexual endocrine disrupting compounds, to include all active substances which may affect development or reproduction through effects linked to steroid hormone pharmacology. This widening in scope is supported by 
results reported in ERAs for centralized procedures and in several articles published in the scientific literature, since the current guideline was issued in 2006.

\section{Environmental fate testing}

For ERAs performed according to the current guideline, the results of the OECD TG 308 test are used to determine if a substance proceeds to sediment toxicity testing. However, nearly all pharmaceutical products meet the trigger criterion for shifting to sediment, which means that a sediment ERA needs to be performed. OECD 308 is a lengthy, expensive and resource demanding test generally requiring the use of radiolabelled active substance and besides the trigger criterion for shifting to sediment, all other study endpoints are often not used for the ERA. In the draft guideline revision, the OECD TG 308 aerobic and anaerobic transformation in aquatic sediment systems test is replaced with mandatory sediment toxicity testing in phase II/Tier A and the OECD TG 308 study is only requested for PBT assessment (triggered by log Kow > 4.5).

\section{Trigger for soil assessment}

The soil adsorption coefficient $\left(K_{\mathrm{d}}\right)$ measures the partitioning of a drug substance between soil and water. $K_{\mathrm{d}}$ normalized to the organic carbon content of the soil $\left(K_{\mathrm{oc}}\right)$ is an indicator of the binding capacity of a substance on organic matter of soil and sewage. In the current guideline, soil assessment is only required when the $K_{\mathrm{oc}}$ in sludge is $>10,000 \mathrm{~L} / \mathrm{kg}$. However, substances with lower adsorption affinity may also be present in sludge at high concentrations when their release to sewage treatment plants is high. This has been demonstrated by the presence of high concentrations of some active substances in sludge samples despite them not having a $K_{\mathrm{oc}}>10,000 \mathrm{~L} /$ $\mathrm{kg}$, e.g., carbamazepine, ibuprofen and some antibiotics $[8,9]$. A change to a combined trigger considering both sorption as well as predicted surface water concentration is, therefore, proposed. In this way, some additional substances with a lower $K_{\mathrm{oc}}$, but with a high release in sewage treatment plants will be captured and will need to proceed to soil assessment.

\section{Potential for secondary poisoning (SP)}

Assessment of potential for SP has been introduced into the guideline revision. SP is relevant for compounds that accumulate through the food chain, mainly lipophilic compounds. In the current guideline, a $\log K_{\mathrm{ow}} \geq 3$ triggers a bioconcentration study, but there is no guidance on how to assess and use the results from this fish study for the assessment of secondary poisoning. Guidance is available in other regulatory frameworks (including the veterinary ERA guideline [10], REACH [11], and the water framework directive [12]. The assessment is based on data from the bioconcentration study in fish, together with results from mammalian toxicity studies submitted in common technical document (CTD) Module 4. There is no need for additional experimental work besides the bioconcentration study. In situations where mammalian toxicity data are not available, further assessment can be waived in justified circumstances.

\section{Labeling and risk mitigation}

The guideline revision proposes labeling based on the ERA and aimed at minimizing the discharge of unused medicines into the environment. The draft revised guideline additionally encourages applicants to share details on analytical verification of their active substances in the form of a report on analytical verification on their websites or in a general database, especially for those active substances with a risk to the environment. This information is essential for water managers, who wish to monitor substances of concern.

\section{PBT assessment}

PBT substances are substances which may persist in the environment and bioaccumulate in organisms. Due to their physico-chemical characteristics, prediction of the environmental fate of these substances or their adverse effects occurring over extended periods is not possible. Chronic exposure and long-term cumulative adverse effects may lead to uncertainty when calculating the PEC via established exposure models and/or establishing the predicted no-effect concentration (PNEC) from standard laboratory tests. Risk assessment and PBT assessment have been separated in the revised guideline to emphasize that PBT assessment, which is a hazard assessment, is performed independent of the risk assessment. Because the PBT assessment is a hazard assessment, every active substance should be assessed for its PBT properties regardless of its PEC. Where a risk assessment is based on considering established threshold values for PECs, the PBT assessment is triggered by the outcome of a study on octanol-water partitioning rather than considering environmental loads. The revision advises following the REACH guidance on PBT assessment as closely as possible but provides considerably more detail than the current guideline on PBT assessment. For example, species other than fish (e.g., mussels) can also be used for bioconcentration studies. It should be noted that a lack of accumulation in mammals does not automatically exclude a potential for accumulation in fish and other aquatic species. The criteria for the assessment of $\mathrm{P}, \mathrm{B}$ and $\mathrm{T}$ properties (as specified in REACH Annex XIII) are described. Toxicity criteria are described which would need to be fulfilled for a substance to meets the $\mathrm{T}$ 
criterion. For those substances where a phase II assessment is triggered, sufficient toxicity studies are already available to verify whether the $\mathrm{T}$ criterion is met or not.

\section{Conclusions}

The draft guideline revision is available for a public consultation period ending on June 30, 2019. It aims to provide a more consistent and holistic approach to environmental risk assessment for human medicinal products for both assessors and applicants. The structure of the guideline has been modified so that in the revised guideline, the assessment (Tier A+Tier B) is completed for every environmental compartment. The decision tree approach will clarify which substances require (further) assessment, while the additional technical information included, particularly for PBT and soil assessment, will reduce the need for applicants and assessors to consult guidance documents from other frameworks. Although some additional testing may be necessary for some legacy substances where insufficient data are available, applicants are encouraged to share data in the interest of reduced (animal) testing. This should be accompanied by reduced testing due to tailored assessments and the limitation in the use of the laborious aerobic and anaerobic transformation test.

\section{Abbreviations \\ CTD: common technical document; EAS: endocrine active substance; EMA: European Medicines Agency; ERA: environmental risk assessment; HMP: human medicinal product; PEC: predicted environmental concentration; PNEC: predicted no-effect concentration; PBT: persistent, bioaccumulative and toxic substance; Q \& A: questions and answers; SP: secondary poisoning. \\ Authors' contributions \\ All authors read and approved the final manuscript. \\ Author details \\ ${ }^{1}$ European Medicines Agency (EMA), Amsterdam, The Netherlands. ${ }^{2}$ Federal Institute for Drugs and Medical Devices (BfArM), Bonn, Germany. ${ }^{3}$ Health Products Regulatory Authority (HPRA), Dublin, Ireland. ${ }^{4}$ Department of Bio- science, Aarhus University, Aarhus, Denmark. ${ }^{5}$ Danish Medicinal Agency, Copenhagen, Denmark. ${ }^{6}$ National Institute for Public Health and the Environ- ment (RIVM), Bilthoven, The Netherlands. ${ }^{7}$ Swedish Medical Products Agency, Uppsala, Sweden. ${ }^{8}$ The Norwegian Medicines Agency (NOMA), Oslo, Norway. \\ ${ }^{9}$ Medicines \& Healthcare Products Regulatory Agency (MHRA), London, UK. \\ ${ }^{10}$ German Environment Agency (UBA), Dessau-Roßlau, Germany.}

\section{Acknowledgements}

We thank Drs. Ana Simon-Hidalgo, Milton Bonelli and Julia Climent Fabrega for their valuable suggestions.

\section{Competing interests}

The authors declare that they have no competing interests.
Consent for publication

We give consent for publication.

Ethics approval and consent to participate

Not applicable.

Funding

Own funding was used for writing of manuscript.

\section{Publisher's Note}

Springer Nature remains neutral with regard to jurisdictional claims in published maps and institutional affiliations.

Received: 18 January 2019 Accepted: 20 February 2019

Published online: 11 March 2019

\section{References}

1. Committee for Medicinal Products for Human Use (CHMP), European Medicines Agency. Guideline on the environmental risk assessment of medicinal products for human use Draft. EMEA/CHMP/SWP/4447/00 Rev. 115 November 2018

2. Committee for Medicinal Products for Human Use (CHMP), European Medicines Agency. Guideline on the environmental risk assessment of medicinal products for human use. EMEA/CHMP/SWP/4447/00 corr 101 June 2006

3. Committee for Medicinal Products for Human Use (CHMP), European Medicines Agency. Questions and answers on 'Guideline on the environmental risk assessment of medicinal products for human use'. EMA/ CHMP/SWP/44609/2010, Rev 126 May 2016

4. Committee for Human Medicinal Products (CHMP) Concept paper on the revision of the 'Guideline on the environmental risk assessment of medicinal products for 6 human use' (EMEA/CHMP/SWP/4447/00 corr 2) 28 April 2016

5. DIRECTIVE 2010/63/EU of the European Parliament and of the Council of 22 September 2010 on the protection of animals used for scientific purposes

6. DIRECTIVE 2001/83/EC of the European Parliament and of the Council of 6 November 2001 on the community code relating to medicinal products for human use

7. Eco-PharmacoStewardship (EPS) Pillar 3-Extended environmental risk assessment (eERA) https://www.efpia.eu/media/25278/pillar-3-extendedenvironmental-risk-assessment-eera.pdf. Accessed 10 Dec 2018

8. Martín J, Camacho-Muñoz D, Santos JL, Aparicio I, Alonso E (2012) Occurrence of pharmaceutical compounds in wastewater and sludge from wastewater treatment plants: removal and ecotoxicological impact of wastewater discharges and sludge disposal. J Hazard Mater 239-240:40

9. Martín J, Santos JL, Aparicio I, Alonso E (2015) Pharmaceutically active compounds in sludge stabilization treatments: anaerobic and aerobic digestion, wastewater stabilization ponds and composting. Sci Total Environ 503-504:97

10. Guideline on environmental impact assessment for veterinary medicinal products in support of the VICH guidelines GL6 and GL38. EMA/CVMP/ ERA/418282/2005

11. ECHA Guidance on information requirements and chemical safety assessment. Chapter R.16: environmental exposure assessment. Version 3.0 February 2016

12. EC (European Communities) (2011) Technical guidance for deriving environmental quality standards. 1255 common implementation strategy for the water framework directive (2000/60/EC). Guidance 1256 Document No. 27, Technical Report-2011-055

Availability of data and materials

Not applicable. 